III Russian National Conference

"Information Technology in Biodiversity Research"

\title{
Study of the vascular plants diversity in the surroundings of the Kytalyk Research Station (Arctic Yakutia)
}

\author{
Olga V. Khitun*(a), Tatiana M. Koroleva (a), Vladislav V. Petrovsky (a), \\ Maitane Iturrate-Garcia (b), Gabriela Schaepman-Strub (b)
}

(a) Komarov Botanical Institute RAS

2 Prof. Popov Street, 197376 Saint-Petersburg, Russia

(b) University of Zurich, 190 Winterthurerstrasse, 8057 Zurich, Switzerland

\begin{abstract}
The local flora (LF) surrounding the research station at the Kytalyk Resourse Reserve was studied in detail and an annotated checklist is presented. Species composition of Kytalyk area flora is compared with the LF in three neighboring areas in the lower reaches of the Indigirka River. These localities had differing topography, therefore their species richness varied. The LF of the Kytalyk Research Station area numbered 131 vascular plant species from 66 genera and 28 families. Low species richness is explained by the prevalent flat and homogenous lowland topography. We found new and the northernmost in this region locations of several boreal and hypoarctic species (Moehringia lateriflora, Salix myrtilloides, Alnus fruticosa, Polygonum tripterocarpum, Orthilia obtusata, Pedicularis pennellii, Utricularia intermedia, U. minor, Triglochin maritima). The taxonomic structure of all four floras is similar, however in the Kytalyk area the Fabaceae, Lycopodiacea and Gentianaceae families were not found, whereas the proportion of Salicaceae, Caryophyllaceae and Scrophulariaceae was higher than in the other LF. We estimated species behavior with landscape activeness scores. Non-active stenotopic species with low abundance comprise $42 \%$ of the flora and species with broad-ecological tolerance comprise $18 \%$ of the flora. Phytogeographical latitudinal groups (arctic, hypoarctic and boreal) are almost equally represented in the LF. Geographical and taxonomic structure of the LF confirm its position in the bioclimatic subzone D.
\end{abstract}

Keywords: habitat type, landscape activeness, local flora, rare species, taxonomical and geographical structure

(C) 2020 Olga V. Khitun, Tatiana M. Koroleva, Vladislav V. Petrovsky,

Maitane Iturrate-Garcia, Gabriela Schaepman-Strub

This is an open access article distributed under the terms of the Creative Commons Attribution License (CC BY 4.0), which permits unrestricted use, distribution, and reproduction in any medium, provided the original author and source are credited.

Published by Ural Federal University and peer-reviewed under responsibility of BDI-2020

(III Russian National Conference "Information Technology in Biodiversity Research")

\section{Introduction}

The global problems of biodiversity conservation, the study of the impacts of climate change and the human activities on ecosystems, demand accurate background information, in particular inventories and monitoring of vegetation and flora changes (Walker et al. 2013). The local flora method has been used in the Arctic for more than 70 years. The essence of the method is that a complete assay of vascular plant species composition of a territory of ca. $100 \mathrm{~km}^{2}$ in area is assessed by thoroughly searching in 
all the habitat types present in the region with consistent repetition (Khitun et al. 2016). Following the initiative of B.A. Yurtsev, a network of local floras (LF) was established in the Russian Arctic and this currently includes 318 sites and allows analysis of various floristic parameters (Yurtsev et al. 2001; Khitun et al. 2018). However, the density of this network is uneven.

The flora in the lower reaches of the Indigirka River was poorly investigated. Therefore, in 2013-2014, we carried out surveys in four localities (Fig. 1):

1) surroundings of Chokurdakh village on the left bank of Indigirka $\left(70^{\circ} 39^{\prime} \mathrm{N}\right.$; $\left.147^{\circ} 53^{\prime} \mathrm{E}\right)$

2) surroundings of Kytalyk Research Station on the left bank of the Berelekh River $\left(70^{\circ} 49^{\prime} \mathrm{N} ; 147^{\circ} 48^{\prime} \mathrm{E}\right)$;

3) surroundings of the Burulgin Cape on the right bank of Indigirka $\left(70^{\circ} 47^{\prime} \mathrm{N}\right.$; $\left.148^{\circ} 45^{\prime} \mathrm{E}\right)$

4) surroundings of Russkoe Ustje settlement in the Indigirka delta $\left(71^{\circ} 07^{\prime} \mathrm{N}\right.$; $\left.149^{\circ} 05^{\prime} \mathrm{E}\right)$.

Previous surveys in the surroundings of Chokurdakh (Boch and Tzareva 1974; Egorova et al. 1991), did not aim to assess the complete LF. Field work by Tatiana Koroleva in 2013-2014 expanded the list of species from 133-167 (Boch and Tzareva 1974; Egorova et al. 1991) to 222. Annotated species check-list from Chokurdakh was recently published (Koroleva et al. 2019). Other LF assays are not yet published.

In this paper, we describe the LF of the surroundings of the Kytalyk Research Station (hereafter "Kytalyk"), present a checklist of the vascular species and compare this LF with three others. Intensive ecological and hydrological research is ongoing at the station (van Huissteden et al. 2005; Schirrmeister et al. 2012; de Klerk et al. 2014; Iturrate-Garcia et al. 2016; and many others), however, many of these works provide only very brief characteristic of vegetation and in some of them we found misidentified species. There were no floristic inventory in this area. An anonymous list of species, circulating at the station, numbers about 40 species and contains only the most notable and dominant species. Therefore, it is important to publish the complete list of species for this area. Olga Khitun, M. Iturrate-Garcia and G. Schaepman-Strub carried out floristic survey in the summer of 2013. Earlier, we discussed theoretical nuances of the study of this LF (Khitun et al. 2014). The area is very homogenous in topography; lacking many habitats, present in other studied localities. However, these lowland wetlands are spread throughout the area, which is much larger than the standard sampling size of LF, so there is no constraint to consider them as comparable with other LFs.

The aim of this paper is to describe the diversity and the specific of vascular plant flora of Kytalyk, show its spatial differentiation, its taxonomic, geographic and ecological structure and to compare with neighboring LFs.

\section{Material and methods}

Study area

The Kytalyk Research Station is located within the Kytalyk Resource Reserve. The reserve was organized in Allaikhovskii district by the order of Yakutian government in 1996 with the financial support from the WWF. The main aim of the Resource Reserve is wetlands conservation and preservation of Yakutian populations of endangered avian species, such as Siberian Crane, Brent Goose, Spectacled Eider, Steller's Eider, Ross's Gull, etc. The Reserve consists of coastal part and the Berelekh 
River valley. We studied the flora of the latter. The Kytalyk station is located on the left bank of the Berelekh River, which is a tributary of Indigirka (Fig. 1).
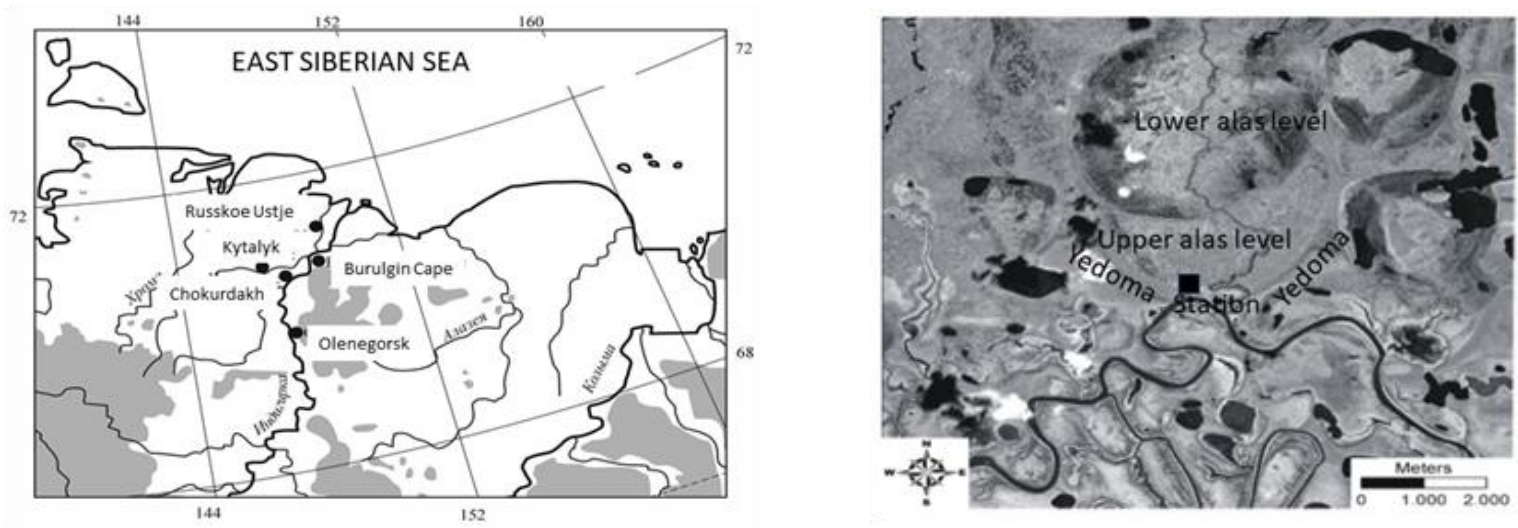

Figure 1. Location of the studied local floras (left) and satellite image of the surroundings of the Kytalyk Research Station (right).

The climate of the Indigirka Lowland is characterized by a high degree of continentality (Gavrilova 1998). Climate data for this area according to the site "Pogoda i klimat" (http://www.pogodaiklimat.ru/history/21946.htm) is only collected at the Chokurdakh weather station, WMO 21946. For the period 1996 to 2013, the mean annual air temperature was $-14.3^{\circ} \mathrm{C}$, the mean January temperature was $-32.9^{\circ} \mathrm{C}$ and the mean temperature of the warmest month, July was $+10.5^{\circ} \mathrm{C}$. The annual precipitation normally varies between $150-250 \mathrm{~mm}$. Recent data indicates a slight warming trend (Koroleva et al. 2019). All four sites are situated in the continuous permafrost zone.

The landscapes in the surroundings of Chokurdah and Burulgin Cape are the most diverse, as outreaches of, respectively, the Allaikhovski and Kondakovski Highlands stretch there, providing greater variation of edaphic conditions. Surroundings of Russkoe Ustje and Kytalyk are monotonous lowlands. The altitude in Kytalyk varies between 3 and $30 \mathrm{~m}$ above sea level. The main landscape subdivisions in Kytalyk are:

1) floodplain and low terraces of the Berelekh River and its tributaries;

2) approximately $5.5 \mathrm{~km}$ in diameter alas depression (i.e. spacious drained lake basin) of the Holocene age with clearly pronounced polygonal structure, numerous thermokarst lakes and solitary pingos; within it two major levels with 1 to $1.5 \mathrm{~m}$ difference can be distinguished;

3) two elongated ridges 20 to $30 \mathrm{~m}$ above sea level height of late Pleistocene Ice Complex deposits, called Yedoma.

On the river terraces thermokarst processes are active. The riverbanks show thaw slumps. More detailed description of the periglacial and thermokarst features in surroundings of the station, is given in Schirrmeister et al. (2012).

According to the Circumpolar Arctic Vegetation Map (CAVM Team 2003), Kytalyk, Chokurdakh, and Cape Burulgin are part of bioclimatic subzone D, i.e. erect dwarf-shrub subzone; Russkoe Ustje lies at the southern border of the subzone C, i.e. hemiprostrate dwarf-shrub subzone.

\section{Methods}

We thoroughly examined the localities by radial transects of various length (up to $10 \mathrm{~km}$ from base camp) aiming to visit all habitat types present in the region, with, if 
possible, at least 5 replicas. We compiled species lists with records on species abundance in different habitats. We distinguished habitat types visually according to topography, hydrology and vegetation (Khitun 1998). Additionally, we established $5 \times 5 \mathrm{~m}$ relevé plots in various habitat types to assess syntaxonomy of vegetation (not covered in this paper). In Kytalyk we studied spatial structure of local flora by distinguishing partial floras (Yurtsev 1987), that is sets of species of various habitat types. Similar work we did previously in the West Siberian Arctic (Khitun 1998). We obtained partial flora of certain habitat type by joining species lists compiled in habitats of this type.

To estimate the behavior of each species within the locality, we use the concept of 'landscape activeness' (Yurtsev 1968). It is a complex index based on three parameters:

1) ecological tolerance, or range of environmental conditions, which species can tolerate, i.e. range of habitats where species occur;

2) abundance;

3) constancy of species occurrence in its habitats.

We estimated ecological tolerance as percentage of habitat types where species occur, e.g. stenotopic species occur in $<25 \%$ of all habitat types; hemi-stenotopic occur in $26-50 \%$ of habitat types, etc. Species got scores according to the table (Table 1), $\mathrm{V}$ is extremely active, IV - very active, III - moderately active, II - low active and I is non-active.

Table 1. Estimation of species activeness scores (modified after Yurtsev 1968).

\begin{tabular}{|c|c|c|c|c|c|c|c|c|c|c|}
\hline Abundance & \multicolumn{10}{|c|}{ Ecological tolerance } \\
\hline & \multicolumn{2}{|c|}{ Eurytopic } & \multicolumn{2}{|c|}{ Hemi-eurytopic } & \multicolumn{2}{|c|}{ Hemi-stenotopic } & \multicolumn{4}{|c|}{ Stenotopic } \\
\hline & & & & & & & Comm & n habitats & Rare 1 & abitats \\
\hline & $\begin{array}{l}\text { Perva- } \\
\text { sive }\end{array}$ & $\begin{array}{c}\text { Spora- } \\
\text { dic }\end{array}$ & $\begin{array}{c}\begin{array}{c}\text { Perva- } \\
\text { sive }\end{array} \\
\end{array}$ & $\begin{array}{l}\text { Spora- } \\
\text { dic }\end{array}$ & $\begin{array}{l}\text { Perva- } \\
\text { sive }\end{array}$ & $\begin{array}{c}\text { Spora- } \\
\text { dic }\end{array}$ & $\begin{array}{c}\text { Cons- } \\
\text { tant }\end{array}$ & $\begin{array}{c}\text { Non- } \\
\text { constant }\end{array}$ & $\begin{array}{c}\text { Cons- } \\
\text { tant }\end{array}$ & $\begin{array}{c}\text { Non- } \\
\text { constant }\end{array}$ \\
\hline Copious & $\mathrm{V}$ & $\mathrm{V}$ & IV & IV & III & II & III & II & II & I \\
\hline Sparse & IV & IV & III & III & III & II & III & $\mathrm{I}$ & $\mathrm{I}$ & $\mathrm{I}$ \\
\hline Solitary & III & II & II & II & II & $\mathrm{I}$ & II & I & $\mathrm{I}$ & $\mathrm{I}$ \\
\hline
\end{tabular}

Determination of species was mainly carried out in the field, however, herbarium vouchers were collected of all the species and these were checked in the laboratory. V.V. Petrovsky verified identification of difficult for determination species. Herbarium specimens are preserved in Komarov Botanical Institute. The nomenclature mainly follows Annotated Checklist of the Panarctic Flora (Elven 2007) as it was prepared by international team of specialists in arctic species, other databases are more focused on southern regions, however in clear cases we updated according to Plants of the World Online (POWO, 2019). All species were assigned in phytogeographical (latitudinal) groups based on their distribution ranges; this work was done previously for LF network (Khitun et al. 2016).

\section{Results}

The species richness of the four LF varies depending on topography of the sites. LF of Chokurdakh and the Burulgin Cape surroundings are the richest (222 and 240 species). Two lowland LF are essentially poorer: we found 150 species in Russkoe Ustje and 131 species in Kytalyk. Their poverty is merely explained by low diversity of 
habitats. We think, that additional 10-15 species can be found in Kytalyk if visit two remote pingos, which we did not reach.

We distinguished 12 habitat types in Kytalyk (Table 2). That is less than in lowland West Siberian Arctic, where 15-22 habitat types were distinguished and the richness of analogous types was somewhat higher there than in Kytalyk (Khitun 1998). Approximately $80 \%$ of the area in Kytalyk is occupied by polygonal tundra-mire complexes in alas depression and $20 \%$ by all other habitat types.

Table 2. Habitat types in Kytalyk and number of species (Nsp) in their partial floras.

\begin{tabular}{|c|c|c|}
\hline ID & Habitat type and vegetation in it & Nsp \\
\hline 1 & $\begin{array}{l}\text { The top and gentle slopes of Yedoma ridge, relatively well-drained, mesic, } \\
\text { acidic soils, with earth hummocks and thick vegetation mat; occupied by } \\
\text { tussock tundra with Eriophorum vaginatum, Ledum decumbens, Betula exilis, } \\
\text { Salix pulchra, Calamagrostis holmii. }\end{array}$ & 31 \\
\hline 2 & $\begin{array}{l}\text { Polygons }(7 \times 8,10 \times 12,15 \times 15 \mathrm{~m}) \text { in the high-centered polygonal tundra- } \\
\text { mire complexes, within the upper level of alas depression and on river terraces; } \\
\text { mesic acidic soils with thick peat horizon; with dwarf birch dominated } \\
\text { communities with thick moss mat (Dicranum spp., Polytrichum spp.) }\end{array}$ & 21 \\
\hline 3 & $\begin{array}{l}\text { Oligotrophic wet troughs (depressions) between polygons, present mainly in } \\
\text { alas depression and on lower river terraces, with shallow water, usually with } \\
\text { dense stand of sedges (Eriophorum angustifolium, Carex concolor). }\end{array}$ & 12 \\
\hline 4 & $\begin{array}{l}\text { Rims of polygons in low centered polygonal complexes, present mainly at the } \\
\text { lower level of alas depression; wet, with herbaceous-dwarf-shrub-moss } \\
\text { (predominantly Sphagnum spp.) communities. }\end{array}$ & 32 \\
\hline 5 & $\begin{array}{l}\text { Ponds-like central depressions of polygons in low-centered polygonal complex, } \\
\text { mainly at the lower alas level; occupied by mires with sedges (Carex } \\
\text { chordorrhiza, C. rotundata) and Amblystegiaceae-mosses. }\end{array}$ & 28 \\
\hline 6 & $\begin{array}{l}\text { Relatively short }(15-20 \mathrm{~m}) \text {, rather steep }\left(10-12^{\circ}\right) \text { and dry slopes of the ridge } \\
\text { and lower pingos are covered by ericoid dwarf-shrub dominated tundra with } \\
\text { abundance of low willow (Salix glauca) and herbs (Pyrola grandiflora, } \\
\text { Pedicularis capitata, Saxifraga nelsoniana, Bistorta plumosa, Valeriana } \\
\text { capitata) and well developed moss cover. }\end{array}$ & 39 \\
\hline 7 & $\begin{array}{l}\text { Long }(20-30 \mathrm{~m}) \text {, steep }\left(10-12^{\circ}\right) \text {, dry slopes of the pingo. Rare habitat, we } \\
\text { visited only one such pingo; grass-herb-Dryas community without continuous } \\
\text { moss cover. Many herbs and grasses were found only here. }\end{array}$ & 47 \\
\hline 8 & $\begin{array}{l}\text { Eroded cut riverbank, cryogenic erosion around thaw lake, landslides on the } \\
\text { northern side of the pingo with pioneer herbaceous vegetation with Arctagrostis } \\
\text { arundinaceae, Puccinellia borealis, Equisetum arvense, Descurainia } \\
\text { sophioides, Draba juvenilis, Artemisia tilesii. }\end{array}$ & 39 \\
\hline 9 & $\begin{array}{l}\text { Wetlands on the first and second river terraces; some inclination and water flow } \\
\text { is present; pH = ca. 6; Amblystegiaceae-mosses dominate, wet meadows and } \\
\text { sedge mires with many rare species (Triglochin maritima, Carex marina, } \\
\text { C. saxatilis, Juncus castaneus, Silene apetala, Minuartia stricta). }\end{array}$ & 41 \\
\hline 10 & $\begin{array}{l}\text { Floodplain homes two distinct vegetation types: somewhat higher parts with } \\
\text { tall }(2-3 \mathrm{~m}) \text { dense willow thickets with Salix pulchra, Salix lanata ssp. } \\
\text { richardsonii, S. anadyrensis and somewhat lower boggy parts with wet } \\
\text { meadows with Carex concolor or Calamagrostis purpurea dominating. }\end{array}$ & 25 \\
\hline 11 & $\begin{array}{l}\text { Snowbeds at hill foots are very rare habitat, many species were found only here } \\
\text { (Salix polaris, Ranunculus nivalis, } R \text {. pygmaeus) }\end{array}$ & 30 \\
\hline 12 & $\begin{array}{l}\text { Aquatic habitats: low mud banks of the lakes and streams, wet sandbanks of the } \\
\text { river, in the water. Usually few species grow but some of them can form stands } \\
\text { or patches (Arctophila fulva, Ranunculus gmelinii, R. pallasii, Hippuris } \\
\text { lanceolata, Caltha arctica) }\end{array}$ & 12 \\
\hline
\end{tabular}


Annotated list of species found in Kytalyk in the summer of 2013

Local flora "Kytalyk" numbers 131 species from 66 genera and 28 families. Families are assembled in Engler system order, and within families, species are listed in an alphabetic order. Each species name is followed by activeness score (Latin numerals), comments, habitat types IDs as in Table 2 (Arabic numerals), latitudinal phytogeographic subgroup. Abbreviations for latitudinal groups and subgroups are the following:

the arctic group (A) includes three subgroups: A, arctic; MA, metaarctic; AA, arctic-alpine;

the hypoarctic group (HA) includes two subgroups: HA, hypoarctic, HAM, hypoarctic-montane;

the boreal group (B) includes $\mathrm{B}$, boreal and $\mathrm{AB}$, arctic-boreal subgroups.

Fam. Equisetaceae

1. Equisetum arvense L. III. Mostly on eroded surfaces. 7, 8. HA.

2. E. variegatum Schleich. ex F. Weber \& D. Mohr. I. Rare. 9. HAM.

Fam. Sparganiaceae

3. Sparganium hyperboreum Laest. ex Beurl. II. Sporadically in little lakes. 12. AB. Fam. Juncaginaceae

4. Triglochin maritima L. I. Rare. Not reported earlier for this part of the Arctic. 9. AB.

Fam. Poaceae

5. Alopecurus alpinus Vill. subsp. borealis (Trin.) Jurtz. II. Rather common. 7, 8. MA.

6. Arctagrostis arundinaceae (Trin.) Beal. III. Abundant on eroded surfaces. 7, 8. HAM.

7. A. latifolia (R. Br.) Griseb. III. Common, sparse. 1, 2, 6, 7, 8. AA.

8. Arctophila fulva (Trin.) Andersson. II. Rather rare, but abundant. 12. HA.

9. Calamagrostis holmii Lange. IV. Common and rather abundant. 1, 2, 4, 5, 6, 7, 8. MA.

10. C. purpurea (Trin.) Trin. [syn. C. langsdorffii (Link) Trin.]. II. Abundant. 10, 12. AB.

11. Dupontia fisheri R. Br. II. Common, but not abundant. 3, 5, 10. A.

12. Elymus macrourus (Turcz.) Tzvelev. I. Rare, solitary. 7. B.

13. Festuca brachyphylla Schult. et Schult. fil. II. Sporadically. 6, 7, 9. AA.

14. Hierochloe pauciflora R. Br. II. Sporadically. 4, 5. HA.

15. Poa alpigena (Blytt.) Lindm. subsp. alpigena [Syn. Poa pratensis L. subsp. alpigena (Lindm.) Hiitonen]. I. Rare. 7. HAM.

16. P. alpigena subsp. colpodea (Th. Fries) Jurtz. et Petrovsky [Syn. Poa pratensis subsp. colpodea (Th. Fr.) Tzvelev] II. Sporadically. 7, 8. MA.

17. P. arctica R. Br. III. Common, but not abundant. 1, 2, 4, 6, 7, 9, 10. AA.

18. Puccinellia borealis Swallen. I. Rare. 8. A.

19. Trisetum spicatum (L.) K. Richt. I. Rare, solitary. 7. AA.

Fam. Cyperaceae

20. Carex bigelowii Torr. ex Schwein. subsp. arctisibirica (Jurtz.) A. et D. Löve. IV. Common and rather abundant. 1, 2, 6, 7. MA.

21. C. concolor R. Br. [Syn. Carex aquatilis Wahlenb. subsp. stans (Drej.) Hult.].

IV. Very common and abundant species. 1, 3, 4, 5, 9, 12. MA

22. C. chordorrhiza Ehrh. II. Sporadically, can be abundant. 5, 9. AB.

23. C. juncella Th. Fries. I. Found once. 12. B.

24. C. marina Dew. [syn. C. amblyorhyncha V. Krecz.]. I. Rare. Solitary. 9. AA.

25. C. rariflora (Wahlenb.) Smith. I. Rare, not abundant. 9. MA.

26. C. rotundata Wahlenb. II. Rather common. 5, 9. HAM. 
Olga V. Khitun, Tatiana M. Koroleva, Vladislav V. Petrovsky, Maitane Iturrate-Garcia, Gabriela Schaepman-Strub / Proceedings BDI-2020

27. C. saxatilis L. subsp. laxa (Trautv.) Kalela. I. Very rare. 9. HAM.

28. C. vaginata Tausch var. vaginata I. Rare. 6. HAM.

29. Eriophorum angustifolium Honck. IV. Common, abundant. 3, 5, 10, 12. AB.

30. E. brachyantherum Trautv. \& C.A. Mey. I. Very rare, solitary. 9. HAM.

31. E. $\times$ medium Andersson. I. Rare. 5. HAM.

32. Eriophorum russeolum Fr. ex Hartm. subsp. leiocarpum M.S. Novos. II. Scattered. 3, 5. HA.

33. E. scheuchzeri Hoppe. I. Rare. 8. AA.

34. E. vaginatum L. IV. Common, abundant. 1, 2, 4, 6. AB.

Fam. Juncaceae

35. Juncus castaneus Smith. I. Very rare. 9. HAM.

36. J. triglumis L. I. Rare. 9. AA.

37. Luzula cofusa Lindb. II. Rather common.1, 2, 6, 7. AA.

38. L. nivalis (Laest.) Spreng. I. Rare. 6, 7. AA.

39. L. tundricola Gorodk. ex V. Vassil. I. Rather rare, solitary. 6, 7. MA.

40. L. wahlenbergii Rupr. II. Common, solitary. 3, 4. HA

Fam. Tofieldiaceae

41. Tofieldia coccinea Richardson. II. Scattered. 1, 6, 7. AA.

Fam. Salicaceae

42. Salix boganidensis Trautv. I. Very rare. 6. HA.

43. S. fuscescens Andersson. II. Common. 3, 4. AB.

44. S. glauca L. III. Common, abundant on slopes. 1, 2, 6, 7, 8, 10. HAM

45. S. hastata L. II. Rather rare. 8, 11. AB.

46. S. myrtilloides L. I. Rare. 4. B.

47. S. pulchra Cham. IV. Common, often abundant. 1, 2, 3, 4, 6, 7, 8, 9, 10. HA.

48. S. reptans Rupr. I. Rare. 7. MA.

49. Salix richardsonii Hook. II. Rather common. 10. HA.

Fam. Betulaceae

50. Alnus alnobetula (Ehrh.) K. Koch subsp. fruticosa (Rupr.) Raus. [Alnus

fruticosa Rupr.]. I. Found once. Probably introduced by humans. 6. AB.

51. Betula nana subsp. exilis (Sukaczev) Hultén]. V. Everywhere, except 12. HA.

Fam. Polygonaceae

52. Bistorta plumosa (Small.) Greene. II. Rather common. 1, 6, 7. MA.

53. B. vivipara (L.) S.F. Gray. I. Rather rare. 7, 9. HAM.

54. Aconogonon tripterocarpum (A. Gray) Hara. I. Found once. 6. HA.

55. Rumex arcticus Trautv. II. Rather common, solitary. 4, 9, 11. HA.

Fam. Caryophyllaceae

56. Cerastium jenisejense Hult. I. Rare. 8, 11. HAM.

57. C. maximum L. II. Rare. 7. AB.

58. C. regelii Ostenf. I. Rare. 8. A.

59. Minuartia rubella (Wahlenb.) Hiern. I. Found once. 7. AA.

60. M. stricta (Sw.) Hiern. I. Found once. 9. HAM.

61. Moehringia lateriflora (L.) Fenzl. I. Rare. 10. AB.

62. Silene uralensis (Rupr.) Bocquet. [Syn. Gastrolychnis apetala (L.) Tolm. et Kozhan.] I. Rare. 9. AA.

63. Stellaria ciliatocepala Trautv. II. Rather common, solitary. 1, 6, 7, 8. HA.

64. S. crassifolia Ehrh. II. Rather rare. 9, 12. AB.

65. S. peduncularis Bunge. I. Rare. 8. AB.

66. S. edwardsii R. Br. I. Found once. 8. MA.

Fam. Ranunculaceae

67. Caltha arctica R. Br. II. Rather common. 3, 10, 12. MA. 
Olga V. Khitun, Tatiana M. Koroleva, Vladislav V. Petrovsky, Maitane Iturrate-Garcia, Gabriela Schaepman-Strub / Proceedings BDI-2020

68. C. palustris L. II. Rather rare. 5, 12. B.

69. Delphinium chamissonis G. Pritz. ex Walp. II. Rather common. 7, 8. MA.

70. Ranunculus arcticus Richardson [Syn. R. pedatifidus subsp. affinis (R. Br.)

Hult.]. I. Rare. 7, 9. MA.

71. . gmelinii DC. II. Sporadically, can be abundant. 12. AB.

72. R. lapponicus L. II. Sporadically. 4. AB.

73. $R$. nivalis L. I. Very rare. 11 . MA.

74. R. pallasii Schlecht. II. Sporadically. 5, 12. A.

75. $R$. pygmaeus Wahlenb. I. Very rare. 11. A.

Fam. Papaveraceae

76. Papaver lapponicum (Tolm.) Nordh. subsp. orientale Tolm. I. Very rare. 7. MA.

Fam. Brassicaceae

77. Cardamine bellidifolia L. I. Found once. 8. AA.

78 C. pratensis L. subsp. angustifolia (Hook.) O.E. Schulz. II. Sporadically. 9, 10. HA.

79. Cochlearia arctica Schlecht. I. Found once. 8. A.

80. Draba hirta L. I. Rather rare. 7, 8. HAM.

81. D. juvenilis Kom. II. More common than previous. 7, 8. HA.

82. D. nivalis Lilj. I. Found once. 7. A.

83. Descurainia sophioides (Fisch. ex Hook) O.E. Schulz. I. Rare. 8. HA.

84. Eutrema edwardsii R. Br. I. Rare. 6. AA.

85. Parrya nudicaulis (L.) Regel. III. Common, not abundant. 1, 6, 7, 8. AA.

Fam. Saxifragaceae

86. Chrysosplenium alternifolium L. subsp. sibiricum (Ser. ex DC.) Hulten. II. Sporadically. 9, 10. AB.

87. C. tetrandrum (Lund ex Malmgr.) Th. Fries. I. Rare. 8. MA.

88. Saxifraga cernua L. III. Sporadically. 4, 5, 8. AA.

89. S. folilosa R. Br. III. Rather common. 3, 4, 5, 10. AA.

90. S. hieracifolia Waldst. et Kit. III. Common. 6, 7, 8, 9, 11. AA

91. S. hirculus L. II. Sporadically. 4, 5, 9. AB.

92. S. nelsoniana D. Don. III. Very common. 1, 4, 6, 7, 8, 9, 10. MA.

93. S. radiata Small. I. Rare. 9. HA.

Fam. Parnassiaceae

94. Parnassia kotzebuei Cham. et Schlech. I. Found once. 11. MA.

Fam. Rosaceae

95. Comarum palustre L. II. Sporadically. 3, 5, 9, 12. AB.

96. Dryas punctata Juz. III. Common. Abundant on slopes. 1, 6, 7. AA.

97. Potentilla stipularis L. I. Very rare. 7. HAM.

98. Rubus chamaemorus L. II. Rather rare. 2, 4. AB.

Fam. Empetraceae

99. Empetrum nigrum subsp. subholarcticum (V.N. Vassil.) Kuvaev. II. Common. 1, 2, 6, 7. AB.

Fam. Onagraceae

100. Epilobium davuricum Fisch. ex Hornem. I. Sporadically. 4, 5, 8, 9. HAM.

101. E. palustre L. II. Rather common. 5, 9, 10, 12. AB.

Fam. Hippuridaceae

102. Hippuris lanceolata Retz. II. Sporadically. 12. HA

103. H. vulgaris L. II. Sporadically. 12. AB.

Fam. Pyrolaceae

104. Orthilia obtusata (Turcz.) H. Hara. II. Sporadically. 1, 6. AB.

105. Pyrola grandiflora Radius. III. Common. 1, 2, 4, 6, 7, 10. AB. 
Fam. Ericaceae

106. Andromeda polifolia L. subsp. pumila V. Vinogr. II. Sporadically. 2, 4, 5, 9. HAM.

107. Arctous alpina (L.) Niedenzu. II. Common. 6, 7. HAM.

108. Cassiope tetragona (L.) D. Don. II. Sporadically.1, 6. MA.

109. Rhododendron tomentosum Harmaja [Syn. Ledum palustre L. subsp. decumbens (Ait.) Hult. IV. Common, abundant. 1, 2, 4, 6, 7. HAM.

110. Vaccinium uliginosum L. subsp. microphyllum Lange. III. Rather common. 1, 2, 6, 7. HAM.

111. V. vitis-idaea L. subsp. minus (Lodd.) Hulten. IV. Common.1, 2, 4, 6, 7, 10. HAM.

Fam. Primulaceae

112. Androsace septentrionalis L. I. Found once. 7. AB.

Fam. Polemoniaceae

113. Polemonium acutiflorum Willd. ex Roem. \& Schult. III. Rather common. 5, $9,10,11,12$. HAM.

Fam. Orobanchaceae

114. Pedicularis capitata Adams. II. Rather rare. 6. MA.

115. P. hirsuta L. II. Rather rare. 6, 7. MA.

116. P. labradorica Wirsing. II. Rather rare. 4. HAM.

117. P. langsdorffii Fisch. ex Steven. I. Rare.6, 7. MA.

118. P. lapponica L. III. Common. 1, 2, 4, 6, 7. HAM.

119. P. pennellii Hultén. II. Rather rare. 3, 4, 5. HA.

120. P. interior (Hultén) Molau \& D.F. Murray [Syn. Pedicularis sudetica subsp. interior (Hultén) Hultén]. III. Common but solitary. 1, 3, 4, 5, 8, 10, 11. HA.

121. P. gymnostachya (Trautv.) A.P. Khokhr. I. Rare. 7. HA.

Fam. Lentibulariaceae

122. Utricularia intermedia Hayne. I. Rare.5, 12. B.

123. U. minor L. I. Rare, but more often than previous. 12. B.

Fam. Adoxaceae

124. Adoxa moschatellina L. II. Sporadically. 7, 8, 10. B.

Fam. Valerianaceae

125. Valeriana capitata Pall. ex Link. III. Scattered. 1, 2, 4, 6, 7, 8, 9, 10. HAM.

Fam. Asteraceae

126. Artemisia tilesii Ledeb. II. Sporadically. 8. MA.

127. Petasites frigidus (L.) Fr. IV. Almost everywhere. 1, 2, 4, 6, 8, 9, 10, 11, 12. AB.

128. Taraxacum longicorne Dahlst. I. Very rare. 7. HA.

129. Tephroseris integrifolia subsp. atropurpurea (Ledeb.) B. Nord. II. Scattered. 1, 2, 6. MA.

130. T. palustris (L.) Rchb. I. Rare. 8. AB.

131. Tripleurospermum hookeri Sch. Bip. II. Sporadically. 8. A.

The set of the top 10 richest by the number of species families is the same in all four studied LFs, however the position of families in this set varies between localities. Naturally, absolute number of species in these families in two poor LFs (Kytalyk and Russkoe Ustje) is less, than in two richer floras (Chokurdakh and Cape Burulgin). That is especially notable in the Poaceae and Cyperaceae families, 35 and 24 species respectively in Chokurdakh, and 24 and 15 species in Kytalyk. Due to rarity of welldrained habitats, xerophylic species of grasses and sedges were totally absent in Kytalyk. For the same reason fam. Asteraceae is poorly represented, it is the third richest in Chokurdakh and only on the $10^{\text {th }}$ place in Kytalyk. Relatively bigger is the portion (but not the absolute number) of fam. Salicaceae, Caryophyllaceae and Scrophulariaceae. 
Leading families in Kytalyk LF are the following: 1) Poaceae - 16 species; 2) Cyperaceae - 15; 3-4) Caryophyllaceae and Salicaceae - 10 species in each; 5) Brassicaceae - 9; 6-8) Saxifragaceae, Ranunculaceae, Scrophulariaceae - 8 species in each; 9-11) Asteraceae, Juncaceae and Ericaceae - 6 species in each. LF Kytalyk differs from neighboring floras by complete absence of families Fabaceae, Lycopodiaceae, Gentianaceae.

We did not find several very common species (Huperzia arctica, Hierochloe alpina, Deschampsia borealis, Parnassia palustris, Astragalus alpinus), which were present in other localities, although we were specifically looking for them. On the other hand, we found new and the northernmost for this region locations of several boreal and hypoarctic species (Moehringia lateriflora, Salix myrtilloides, Alnus fruticosa, Polygonum tripterocarpum, Orthilia obtusata, Pedicularis pennellii, Utricularia intermedia, U. minor, Triglochin maritima). The latter three species were not previously recorded in Yana-Indigirka region at all (Sekretareva 2004) Parnassia kotzebuei and Pedicularis pennellii are included in Yakutian Red Data Book (Dolinin 2001). The latter was absent in Chokurdakh, but other red-listed species (Platanthera oligantha and Vicia macrantha) were found there.

Distribution of species according to the scores of activeness resemble the pattern, which we found in the West Siberian Arctic in subzones E and D (Khitun 1998). Non-active and low active (I and II) species are the most numerous groups (55 and 52 species), they comprise $82 \%$ of total LF. In Kytalyk, species with broader ecological tolerance (III-V) comprise $18 \%$ of total flora, in West Siberian Arctic their proportion is $14 \%$. Only one species (Betula exilis) obtained score V, it was growing practically in all habitats, except aquatic. Interestingly, that although Kytalyk lies only $30 \mathrm{~km} \mathrm{NW}$ from Chokurdakh, behaviour of certain species (which are not rare species in this region) is very different at these localities. For example, Eriophorum vaginatum is one of the most widespread species in Kytalyk, but occurs sporadically in Chokurdakh; the same about Salix pulchra; on opposite, Salix boganidensis and Luzula nivalis are common in Chokurdakh, but rare in Kytalyk. However, really rare species (score I), as a rule, are rare at both sites.

All latitudinal groups are almost equally represented in the flora of Kytalyk, with slightly prevailing arctic group (Table 3 ). This regularity was also found in more diverse floras of Chokurdakh and Burulgin, whereas in LF of Russkoe Ustje, which is located $80 \mathrm{~km}$ to the north from Kytalyk, the arctic group was more notably prevailing.

Table 3. Proportion of species in various latitudinal groups in four local floras.

\begin{tabular}{|l|c|c|c|c|}
\hline $\begin{array}{c}\text { Latitudinal } \\
\text { group }\end{array}$ & Kytalyk & Chokurdakh & Burulgin Cape & Russkoe Ustje \\
\hline Arctic & 40 & 37 & 41 & 49 \\
\hline Hypoarctic & 33 & 32 & 30 & 28 \\
\hline Boreal & 27 & 31 & 29 & 23 \\
\hline
\end{tabular}




\section{Conclusion}

The LF of Kytalyk numbers 131 species, in spite of such paucity, it exhibits all the features typical for its region. It is representative of wet lowland areas in the low reaches of the Indigirka River. We discovered new, and the northernmost for this region, locations of several boreal and hypoarctic species (Moehringia lateriflora, Salix myrtilloides, Alnus fruticosa, Polygonum tripterocarpum, Orthilia obtusata, Pedicularis pennellii, Utricularia intermedia, U. minor, Triglochin maritima). Additionally, we found two endangered species (Parnassia kotzebuei and Pedicularis pennellii). The low diversity of flora is connected with poorly differentiated topography, wetland areas and shortage of slopes and well-drained surfaces. The main part of species diversity in this area is formed by rare stenotopic species with low abundance. Therefore, it is especially actual to maintain regular surveys and monitoring.

In Chokurdakh we carried out our survey 45 years after the first survey by Boch and Tzareva (1974). We recorded the presence of 57 previously unreported species and changes in the frequency of occurrence of 73 species (Koroleva et al. 2019). Analysis of these new species revealed an increased proportion of boreal species among them, besides many new boreal species are anthropochores. In Kytalyk there were no surveys previously. We did not record obviously introduced species. However, we can only speculate about the presence of a few alder shrubs on the ridge slope near the abandoned muskox coral, which was established there in late 1980's. It is possible that seeds arrived with the forage; but it is also possible that the alder spread naturally as there is plenty of alder on the banks of the Indigirka.

According to the species composition of this LF, it belongs to East-Siberian hypoarctic floras. The almost equal proportions of arctic and non-arctic latitudinal groups in this flora confirm its hypoarctic position. In the East Siberian Arctic, in contrast to the West Siberian, bioclimatic subzones distribution is uneven. In some areas, subzone E exists as a very narrow stripe and forest-tundra rapidly transfers into subzone D. That is connected with the high degree of continentality of the climate, which makes it unfavorable for shrubs to grow on watersheds. However, along rivers tall willow and alder thicket spread far to the north. Sharp changes of climatic conditions and vegetation was shown along the $110 \mathrm{~km}$ longitudinal transect in the lower reaches of the Kolyma River, there the zonal vegetation changed from northern forest-tundra via very narrow belts of subzones $\mathrm{E}$ and $\mathrm{D}$ to subzone $\mathrm{C}$ (Koroleva and Petrovsky 2000). Similar changes in the Indigirka lower reaches occurred along a $c a$. $200 \mathrm{~km}$ profile. By comparison: in Yamal and Taymyr, similar zonal changes occur along a $c a .700 \mathrm{~km}$ transect. Therefore, it is difficult to determine the zonal position of any site in the Indigirka-Kolyma region. Analyzing the proportions of latitudinal elements, taking into account that in partial floras of wetlands boreal features are more expressed, and looking at the vegetation on the hills (tussock tundra with abundant ericoid dwarf-shrubs) we confirm the position of this locality in subzone D.

\section{Acknowledgements}

The field study was supported by the University Research Priority Program on Global Change and Biodiversity of the University of Zurich (URPP-GCB). The analytical part was done in the frames of Komarov Botanical Institute research theme AAAA-A19-119030690002-5. 


\section{References}

Boch MS, Tzareva VT (1974) K flore nizoviev reki Indigirki (v predelakh tundrovoi zonu) [About the flora of the lower reaches of the Indigirka River (within the tundra zone]. Botanicheskii Zhurnal 59 (6): 839-849 [in Russian].

CAVM Team (2003). Circumpolar Arctic Vegetation Map. Scale 1:7 500 000. U.S. Fish and Wildlife service, Anchorage, Alaska.

De Klerk P, Teltewskoi A, Theuerkauf M, Joosten H (2014) Vegetation patterns, pollen deposition and distribution of non-pollen palynomorphs in an ice-wedge polygon near Kytalyk (NE Siberia), with some remarks on Arctic pollen morphology. Polar Biology 37: 1393-1412.

Dolinin IN (Ed.) (2001) Krasnaya kniga Respubliki Sakha (Yakutia). Tom 1: Redkie i nakhodyashchiesya pod ugrozoi ischeznoveniya vidy rastenii i gribov [The Red Data Book of the Republic Sakha (Yakutia). Vol. 1: Rare and Endangered Species of Plants and Fungi]. Ministry of Nature Conservation of Republic Sakha (Yakutia), Yakutsk, 256 pp. [in Russian].

Egorova AA, Vasiljeva II, Stepanova NA, Fesko NN (1991) Flora tundrovoi zony Yakutii [Flora of the tundra zone of Yakutia]. Academy of Sciences of the USSR, Siberian Branch, Yakutian Scientific Center, Yakutsk, 186 pp. [in Russian].

Elven R (Ed.) (2007) Annotated Checklist of the Panarctic Flora (PAF) Vascular Plants http://panarcticflora.org [Accessed on 30.07.2020]

Iturrate-Garcia M, O'Brien MJ, Khitun O, Abiven S, Niklaus PA, Schaepman-Strub G (2016) Interactive effects between plant functional types and soil factors on tundra species diversity and community composition. Ecology and Evolution 6 (22): 8126-8137. https://doi.org/10.1002/ece3.2548.

Gavrilova MK (1998) Klimaty kholodnykh regionov zemli [Climates of the Earth's cold regions]. Russian Academy of Science, Siberian Branch, Permafrost Institute, Yakutsk, 206 pp. [in Russian].

Khitun OV (1998) Sravnitelnui analiz lokalnukh i partzialnukh flor v dvukh podzonakh zapadnosibirskoi Arktiki (Tazovskii i Gydanskii poluostrova) [Comparative analysis of local and partial floras in two subzones of West Siberian Arctic (Tazovsky and Gydansky peninsulas)]. In: Yurtsev BA (Ed.) Study of biodiversity by the methods of comparative floristics. Nauka, Saint-Petersburg, 173-201 [in Russian].

Khitun OV, Schaepman-Strub G, Iturrate-Garcia M (2014) Lokalnaya flora okrestnostei statzionara Kytalyk (Respublika Sakha) [Local flora of the surroundings of Kytalyk station (Republic Sakha)]. In: Baranova OG, Litvinskaya SA (Eds) Comparative Floristics: Analysis of plant species diversity. Problems, Prospects. X International Workshop on Comparative Floristics, Krasnodar, April 2014. Kuban State University Press, Krasnodar, 216-222 [in Russian]. 
Khitun OV, Koroleva TM, Chinenko SV, Petrovsky VV, Pospelova EB, Pospelov IN, Zverev AA (2016) Application of local floras for floristic subdivision and monitoring of vascular plants diversity in the Russian Arctic. Arctic Science 2 (3): 103-126. https://doi.org/10.1139/as-2015-0010.

Khitun OV, Chinenko SV, Zverev AA, Koroleva TM, Petrovsky VV, Pospelov IN, Pospelova EB (2018) Gradients of Taxonomic Diversity among Local Floras in the Russian Arctic. In: Tretyakova AS, Veselkin DV (Eds) Fourth International Scientific Conference Ecology and Geography of Plants and Plant Communities. KnE Life Sciences, 80-87. https://doi.org/10.18502/kls.v4i7.3224.

Koroleva TM, Petrovsky VV (2000) Floristicheskie izmeneniya v sostave sosudistykh rastenii na shirotnom profile $\mathrm{v}$ nizovjyakh reki Kolymy [Floristic changes in the vascular species composition along the latitudinal profile in the Kolyma River low reaches]. Botanicheskii Zhurnal 85 (10): 15-38 [in Russian].

Koroleva TM, Gogoleva PA, Petrovsky VV, Zverev AA, Troeva EI (2019) Monitoring lokalnoi flory $\mathrm{v}$ okrestnostyakh poselka Chokurdakh (severo-vostok Yakutii) [Monitoring of local flora in vicinity of Chokurdakh village (Northeastern Yakutia)]. Botanicheskii Zhurnal 104 (9): 32-66 [in Russian].

POWO (2019) Plants of the World Online. Facilitated by the Royal Botanic Gardens, Kew. http://www.plantsoftheworldonline.org [Accessed on 02.10.2020]

Schirrmeister L, Pestryakova L, Wetterich S, Tumskoy V (Eds) (2012) Joint RussianGerman Polygon Project: East Siberia 2011-2014. The expedition Kytalyk 2011. Reports on Polar and Marine Research 653: 1-116.

Sekretareva NA (2004) Sosudistye rasteniya Rossiiskoi Arktiki i sopredelnykh territorii [The Vascular Plants of the Russian Arctic and Adjacent Territories]. KMK-Press, Moscow, 129 pp. [in Russian].

van Huissteden J, Maximov TC, Dolman AJ (2005) High methane flux from an arctic floodplain (Indigirka lowlands, eastern Siberia). Journal of geophysical research 110: G02002. https://doi.org/10.1029/2005JG000010.

Walker DA, Alsos IG, Bay C, Boulanger-Lapointe, Breen AL, Bültmann H, Christensen T, Damgaard C, Daniëls FJA, Hennekens S, Raynolds M, Le Roux PC, Luoto M, Pellissier L, Peet RK, Schmidt NM, Stewart L, Virtanen R, Yoccoz NG, Wisz MS (2013) Rescuing valuable Arctic vegetation data for biodiversity models, ecosystem models and a panarctic vegetation classification. Arctic 66 (1): 133-137. https://doi.org/10.14430/arctic4281.

Yurtsev BA (1968) Flora Suntar-Khayata. [Flora of Suntar-Khayata]. Nauka, Leningrad, 235 pp. [in Russian].

Yurtsev BA (1987) Flora kak bazovoe ponyatie floristiki: soderzhanie, podkhody k izucheniyu [Flora as a basal concept of floristics: content, approaches to study]. In: Yurtsev BA (Ed.) Theoretical and Methodical Problems of Comparative Floristics. Nauka, Leningrad, 13-28 [in Russian]. 
Olga V. Khitun, Tatiana M. Koroleva, Vladislav V. Petrovsky, Maitane Iturrate-Garcia,

Yurtsev BA, Katenin AE, Koroleva TM, Kucherov IB, Petrovsky VV, Rebristaya OV, Sekretareva NA, Khitun OV, Khodachek EA (2001) Opyt sozdaniya seti punktov monitoringa bioraznoobraziya aziatskoi Arktiki na urovne lokalnukh flor: zonalnye trendy [An attempt of a biodiversity monitoring network creation in the Asian Arctic at the level of local flora: zonal trends]. Botanicheskii Zhurnal 86 (9): 1-27 [in Russian]. 